\title{
Effects of Absorption Spectra of Ices on the Ultraviolet Extinction by Interstellar Grains
}

\author{
G. B. Field, R. B. Partridge, and H. Sobel \\ Princeton University \\ Princeton, New Jersey
}

I N SPITE OF MANY YEARS OF RESEARCH ON INTERSTELLAR GRAINS, 1 their composition is still unknown. Evidently it can be found only through the interaction of the grains with radiation, and one is led to the spectroscopic study of the solids composing the grains. If discrete lines, bands, or other spectral features can be discovered, they may be of great importance in the chemical analysis of the grains. So far, data of this type include the diffuse interstellar lines, the optical lines of $\mathrm{CH}$, $\mathrm{CH}^{+}$, and $\mathrm{CN}$ (almost certainly once part of the grains), the radio lines of $\mathrm{OH}$, and the distinct "kink" or curvature in the optical extinction curve (see paper by Nandy in present compilation), which may reflect spectral variations in refractive index and hence indicate a specific material.

The advent of ultraviolet and infrared techniques suggests that consideration be given to new features to be expected in those regions. In the present paper is presented a calculation of features to be expected in the 1000 - to $2000-\AA$ range for particles of ice. Although the calculation is crude and almost certainly in error at certain points, it serves as an example of any case in which photons of 5 to 10 electron volts can cause internal transitions which profoundly affect the optical properties at wavelengths specific for the material. Other investigators will certainly find such effects for other materials.

While this work was in progress, a similar investigation which also included observational data was being carried out for the infrared. (See ref. 1.) By an examination of the 3.1- $\mu$ line of $\mathrm{H}_{2} \mathrm{O}$ ice in the spectrum of $\mu$ Cep, the authors of reference 1 concluded that lack of a detectable line implies that less than 25 percent of the mass of the interstellar grains between the Sun and that star is $\mathrm{H}_{2} \mathrm{O}$, compared with the expected 64 percent (fig. 1). This seems to be the first definitive indica- 
TEMPERATURE

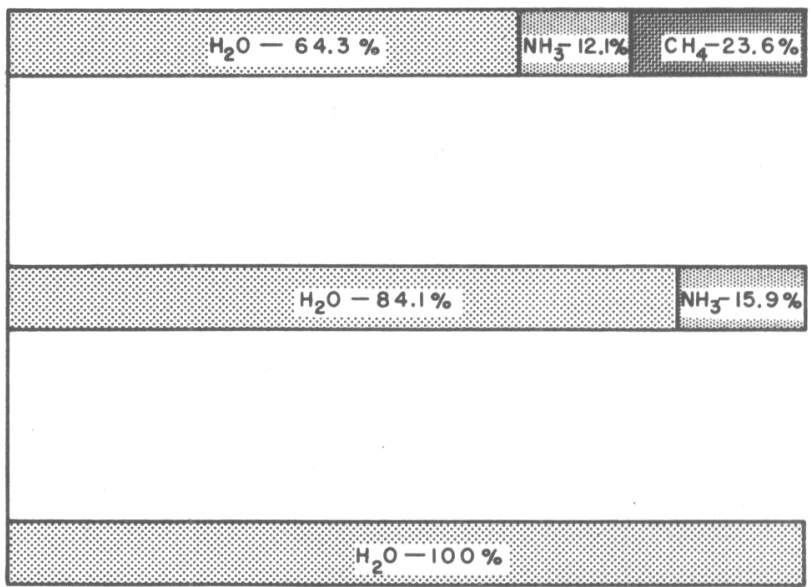

$\mathrm{T}<19^{\circ} \mathrm{K}$

$19^{\circ} \mathrm{K}<\mathrm{T}<56^{\circ} \mathrm{K}$

$56^{\circ} \mathrm{K}<\mathrm{T}<94^{\circ} \mathrm{K}$

\section{PERCENT BY MASS}

FIGURE 1. - Chemical composition of grains. Cosmic abundance ratios assumed for nonevaporated components. The three chemical compositions used were water, ammonia, and methane in cosmic proportions, as modified by evaporation of methane and am. monia at the indicated temperatures.

tion that grains predominantly of ice may not be responsible for the optical extinction and is an example of the power of the spectroscopic method. This early quantitative result should not be taken too literally, however. For example, an alternative calculation of the mass of ice grains required to explain the optical extinction of $\mu \mathrm{Cep}$, based on an exponential distribution of radii with $\bar{a}=0.05 \mu$, is somewhat smaller than was assumed in reference 1 , with the result that the upper limit on $\mathrm{H}_{2} \mathrm{O}$ is revised upward to 45 percent.

\section{CALCULATIONS}

The effects studied here concern the dissociation continua of ices of water, ammonia, and methane discussed in reference 2. Figure 2 shows the values of $n^{\prime}$ (imaginary part of refractive index) for the pure ices implied by their measurements. These values of $n^{\prime}$ were weighted in accordance with the mixtures of figure 1 , which were derived on the assumptions of complete binding of $\mathrm{C}, \mathrm{N}$, and $\mathrm{O}$ in the form of $\mathrm{CH}_{4}$, $\mathrm{NH}_{3}$, and $\mathrm{H}_{2} \mathrm{O}$ in proportion to cosmic abundance, with successively less volatile components evaporating as the temperature was raised in accordance with the equilibrium calculations of reference 3 . These mixtures were taken as representative of variations one might encounter successively closer to an early star (see paper by O'Dell in present compilation for a discussion of evaporation). 


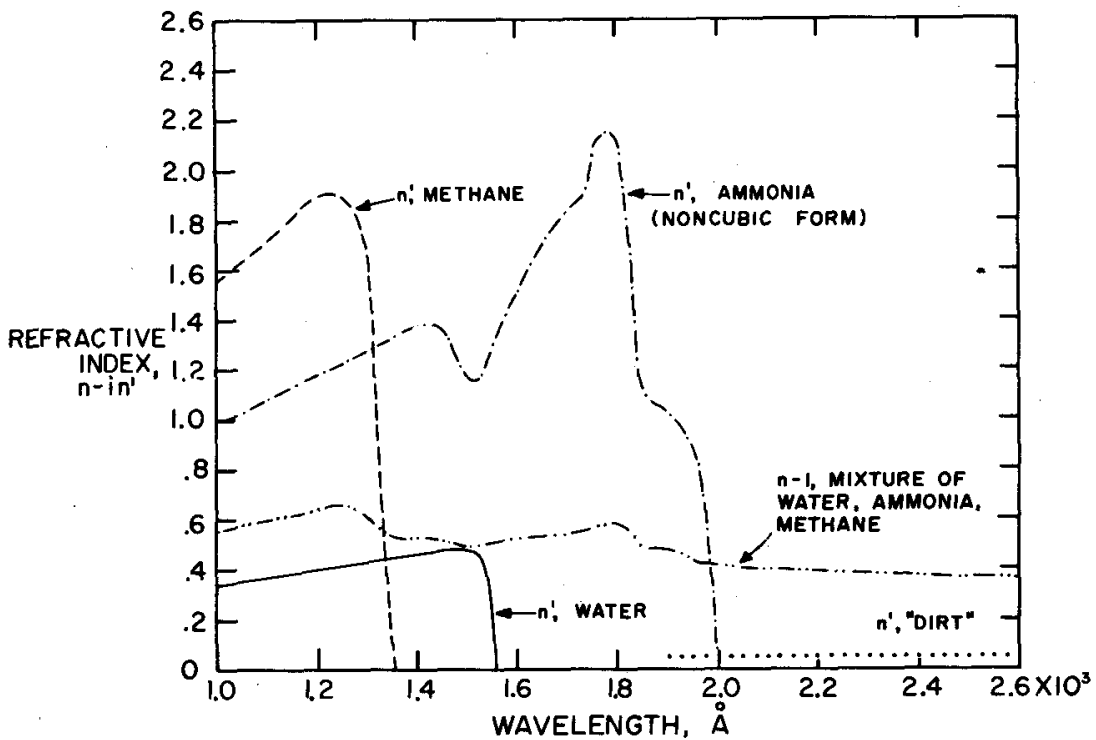

FigUre 2.-Laboratory data on refractive index in the ultraviolet. Note distinct absorption edges for each material. Calculation of $n-1$ was made by weighting approximate expressions representing extrapolations of data in the visible. (From ref. 2.)

It can be seen in figure 2 that in each case $n^{\prime}$ rises sharply at the absorption edge, which occurs for water at $1570 \AA$, for ammonia at $1990 \AA$, and for methane at $1350 \AA$. Rapid changes of $n$ (the real part) are anticipated in the neighborhood of these limits, but these have apparently not been measured and only estimates can be made. Estimates were made as explained in the appendix, where it is shown that for water (which dominates the situation, see fig. 1) one can obtain a better approximation than was used by application of the Kramers-Kronig dispersion relation. The values of $n-1$ in figure 1 , without marked rapid changes, were used for the case of the mixture of water, ammonia, and methane; these values were typical of those used in the other cases. Hence, the spectral changes in the extinction found are due primarily to $n^{\prime}$ and not to $n$. The value of 0.05 was assumed for $n^{\prime}$ for values of the wavelength greater than 1.9 .

An exponential distribution of particle radii obtained from reference 4 was used. Physical reasons are given in reference 4 for such a distribution, and van de Hulst has showed that this distribution gives analytic results for the equation

$$
\bar{Q}(\lambda)=\frac{1}{2 \bar{a}^{3}} \int_{0}^{\infty} \mathrm{e}^{-a / \bar{a}} a^{2} Q(a / \lambda) \mathrm{d} a
$$


in the asymptotic approximation $n-1, n^{\prime} \rightarrow 0$; namely

$$
\frac{1}{2} \bar{Q}=1-\frac{(1+\bar{\rho} \tan \beta)^{2}-\bar{\rho}^{2}}{\left[(1+\bar{\rho} \tan \beta)^{2}+\bar{\rho}^{2}\right]^{2}}
$$

where $Q$ is the extinction efficiency, $\bar{\rho}=\frac{4 \pi \bar{a}(n-1)}{\lambda}$, and $\tan \beta=\frac{n^{\prime}}{n-1}$. It may be shown that equation (1) gives exact results (agrees with Mie theory) in both the limits $\bar{\rho}=0$ and $\bar{\rho}=\infty$ for $\tan \beta>0.05$. Equation (2) for a typical case, $\tan \beta=0.17$, was compared with the exact results of reference 5 averaged according to equation (1), and the maximum difference was 13 percent. Clearly in our cases larger errors are to be expected because $n-1$ and $n^{\prime}$ become large, but the simplicity of this procedure outweighs the modest errors incurred.

\section{RESULTS}

Calculations were carried out for the three mixtures for values of $\bar{a}$ of $0.01 \mu, 0.03 \mu, 0.1 \mu, 0.3 \mu$, and $1.0 \mu$. It was found that the groundbased measurements of extinction summarized in reference 6 could best be fitted with $0.05 \mu$. This fit was good to 5 percent probable error, unexpectedly good for a one-parameter calculation. This should not be considered an indication that the actual particles have the assumed properties, but rather, that if the distribution and materials are those

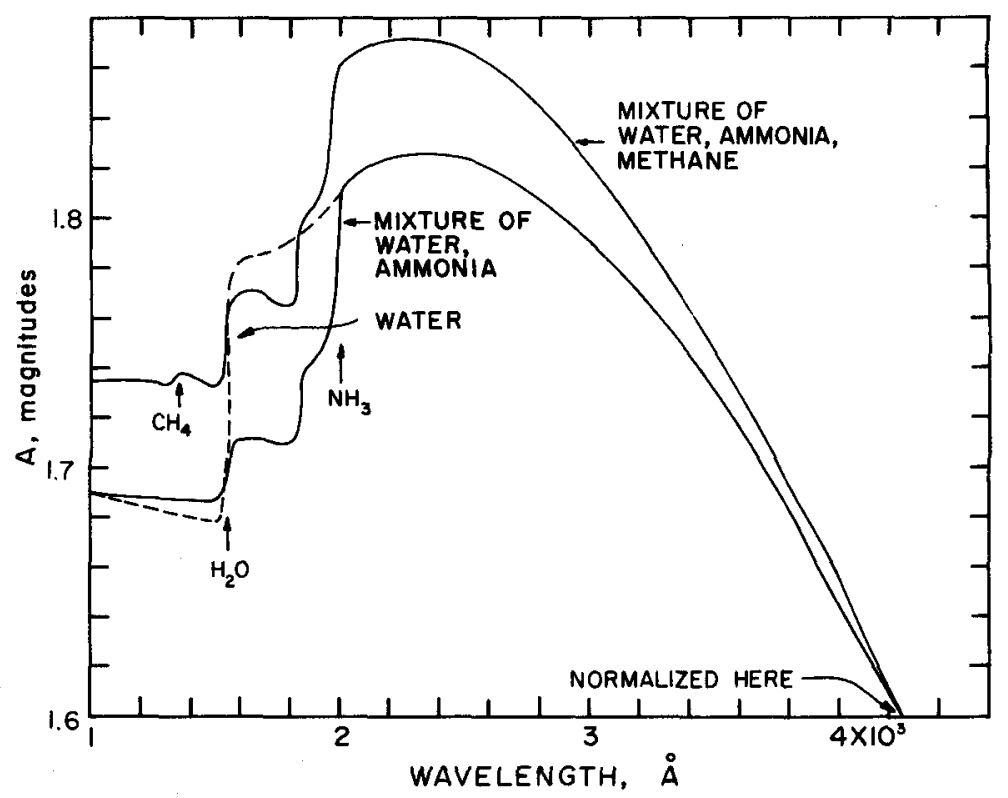

FIGURE 3.-Ultraviolet extinction in magnitudes normalized to 1.6 in the photographic for an exponential particle size distribution with $\bar{a}=1000 \AA$. 


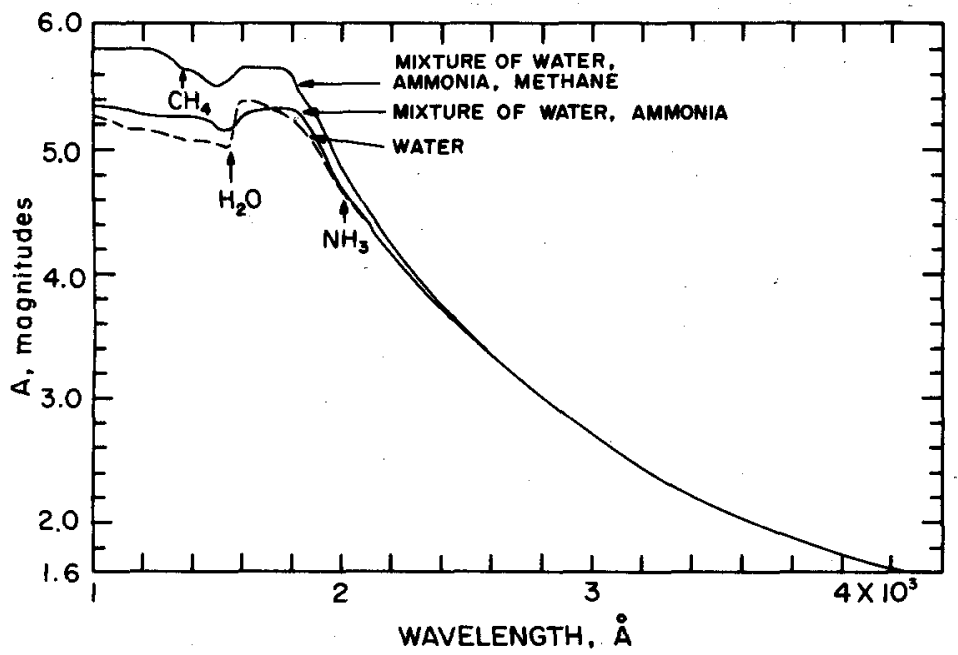

Figure 4. - Ultraviolet extinction in magnitudes normalized to 1.6 in the photographic for an exponential particle size distribution with $a=300 \mathrm{~A}$.

assumed, $\vec{a}$ cannot be very different from $0.05 \mu$. This value is considerably smaller than the mean of the Oort-van de Hulst distribution, $0.19 \mu$. (See ref. 3.) Such a change is obviously indicated by the large increases over van de Hulst's curve at $2200 \AA$ and $2600 \AA$ found in reference 6 .

The results of interest are therefore those for $0.10 \mu$ and $0.03 \mu$, which are shown in figures 3 and 4, normalized to 1.6 magnitudes at $4250 \AA$ : It is seen that the $0.1 \cdot \mu$ curves increase rather slowly from the photographic value in contradiction with the observation at $2200 \AA$ and $2600 \AA$, as explained previously. The effect of interest is the decrease in extinction ahead of the three characteristic limits. This effect, amounting to 5 percent for water and less for the other components, is surprising at first. The explanation is to be found in the fact that at $1550 \AA$, for example, $\bar{\rho} \simeq 6$, so that the grains are larger than the wavelength. The curves on page 28 of reference 3 show that, for large particles of a certain size range, increasing the value of $n^{\prime}$ can decrease the total extinction. This effect is explained as due to a decrease in the amplitude of the rays transmitted by the grain, with the accompanying interference effects which lead to large scattering.

This effect persists with $0.03-\mu$ grains, which more nearly match the observed extinction in the near ultraviolet. Here the effects are percentagewise of the same order, but numerically larger. For an unknown reason, the effect of ammonia is to flatten the otherwise rising curve, while water and methane show abrupt decreases as before.

A value for $\bar{a}$ of $0.01 \mu$ was used to obtain the results shown in figure 5 ; however, large numbers of such particles are probably not present. 


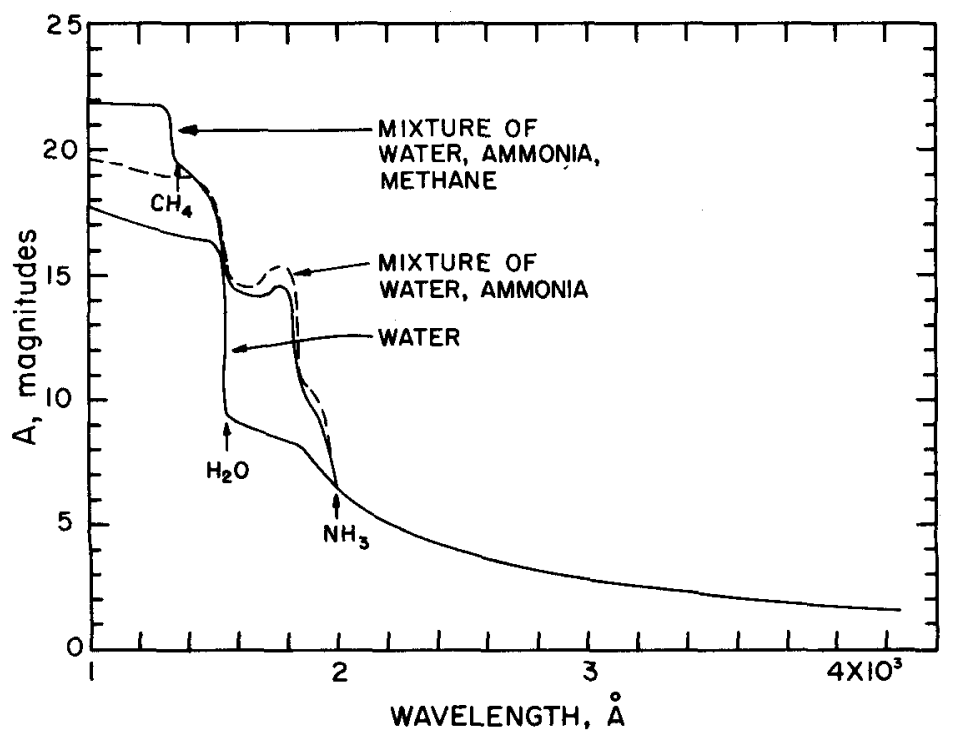

FIGURE 5. - Ultraviolet extinction in magnitudes normalized to 1.6 in the photographic for an exponential particle size distribution with $\bar{a}=100 \AA$.

For these small particles the true absorption is dominant, as predicted by van de Hulst, for $\bar{\rho}<1$. The dramatic increases in extinction amount to 7,9 , and 2 magnitudes for water, ammonia, and methane, respectively.

\section{CONCLUSIONS}

In each case effects are seen which are characteristic of the three assumed components; whether or not these are detectable is unknown. At least one can say that if early-type stars with typical photographic extinctions can be seen at all in these wavelengths, then ice particles with $a=0.01 \mu$ are quite rare, but this fact is already stıongly indicated by the optical data. The abrupt flattening of the curves for $\bar{a}=0.03 \mu$ may possibly become measurable as techniques improve (for such measurements, see the paper by Stecher in the present compilation), and the details, combined with improved versions of the calculation described here, should lead to quantitative estimates of abundances. Present estimates of $\bar{a}$ suggest that rocket observers should not anticipate being hindered by a drastic increase in extinction in the range from $1000 \AA$ to $2000 \AA$, as suggested by the data in figure 1 . Rather, because of van de Hulst's effect, the extrapolation of the curve of reference 6 may well lead to an overestimate of the extinction in this region. 


\section{APPENDIX}

\section{Evaluation of $n$ From $n$ ' by Using Dispersion Relations}

The linear absorption coefficient $\alpha$, related to $n$ by $n^{\prime}=\lambda \alpha / 4 \pi$, where $\lambda$ is the vacuum wavelength, has been measured in reference 2 . The Kramers-Kronig dispersion relation ${ }^{\circ}$ an be written

$$
\epsilon_{1}-1=\frac{2}{\pi} P \int_{\omega_{w}=0}^{\infty} \frac{\nu_{*} \epsilon_{2}\left(\nu_{*}\right) \mathrm{d} \nu_{*}}{\nu_{*}^{2}-\nu^{2}}
$$

where the complex dielectric constant $\epsilon_{1}-\mathrm{i} \epsilon_{2}$ is the square of the complex refractive index $n-i n^{\prime}$. Substitution of $\epsilon_{1}=n^{2}-\left(n^{\prime}\right)^{2}$ and $\epsilon_{2}=2 n n^{\prime}$ $=\lambda \alpha n / 2 \pi$ into equation $(\mathrm{Al})$ yields

$$
n^{2}=\left(n^{\prime}\right)^{2}+\left[1+\frac{c}{\pi^{2}} P \int_{\nu_{*}=0}^{\infty} \frac{n\left(\nu_{*}\right) \alpha\left(\nu_{*}\right) \mathrm{d} \nu_{*}}{\nu_{*}^{2}-\nu^{2}}\right],
$$

which is a nontrivial integral equation for $n$ if $n^{\prime}$ (and hence $\alpha$ ) are given for all frequencies. The method of crude approximation on which values of $n$ in figure 2 are based is simply to equate the expression in brackets to the observed value of $n^{2}$ down to the shortest wavelength for which it has been determined (which is longer than the absorption limit, so that $n^{\prime}=0$ ). Ahead of the absorption limit, $n$ was approximated by

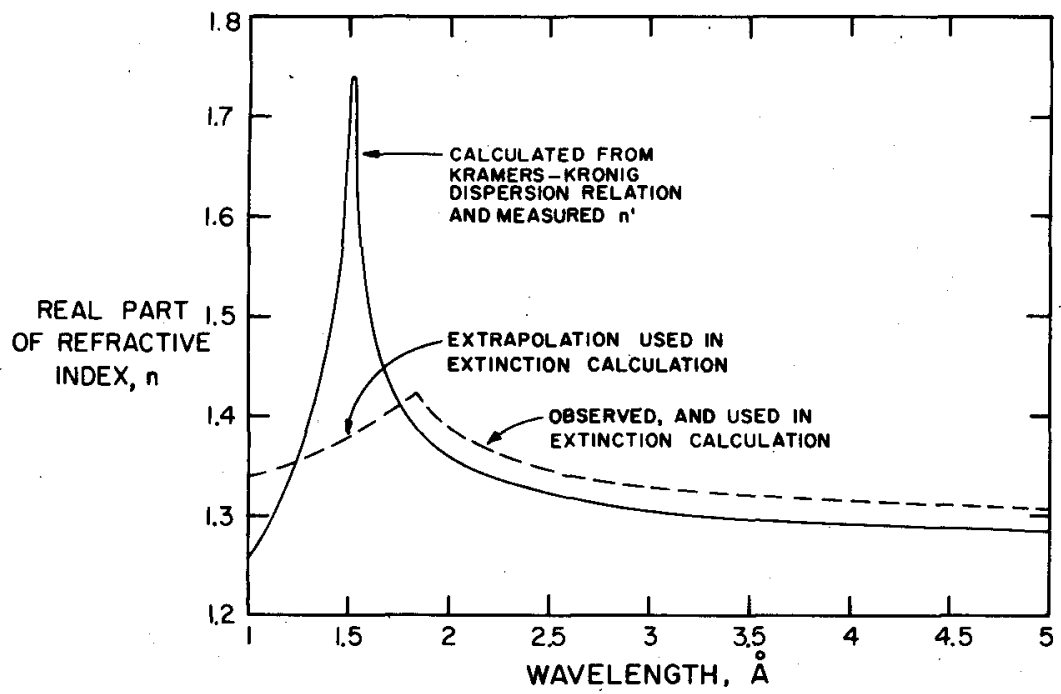

FiguRE 6. - Comparison of values of $n$ for ice as observed in the laboratory and extrapolated for use in present paper (dashed line) with those calculated from observed values of $n^{\prime}$ (fig. 2) based on Kramers-Kronig dispersion relation. The agreement is complete above $1800 \AA$ if a slight adjustment factor in $\alpha_{0}$ is introduced, suggesting that the peak is probably real, and should be used in more accurate calculations. 
$n\}$, the optical value, and this curve was extrapolated to meet the observed one. The results for pure water are represented by the dashed line in figure 6 . Some increase at the absorption limit is introduced by the term $\left(n^{\prime}\right)^{2}$ in equation (A2).

A better approximation is obtained by evaluating the integral with some assumed value of $n$ under the integral sign. A consistent first approximation if $n^{\prime}$ is small is evidently to take $n=1$ under the integral. It was found that $\alpha$ for water could be represented by

$$
\begin{aligned}
\alpha=\alpha_{0} & \nu>\nu_{0} \\
\alpha=\alpha_{0} \exp \left[\left(\nu-\nu_{0}\right) / \Delta \nu\right] & \nu<\nu_{0}
\end{aligned}
$$

with $\alpha_{0}=4.0 \times 10^{5} / \mathrm{cm}^{1}, \quad \lambda_{0}=c \nu_{0}=1.57 \times 10^{-5} \mathrm{~cm}$, and $\Delta \lambda=\lambda_{0}^{2} \Delta \nu / c$ $=2.3 \times 10^{-7} \mathrm{~cm}$. With such a representation, equation (A2) can be calculated analytically to obtain

$$
n^{2}=\left(n^{\prime}\right)^{2}+1+\frac{\lambda_{0} \alpha_{0}}{2 \pi}\left(x ; x_{0}\right)
$$

where $x=\nu / \Delta \nu$ and $x_{0}=\nu_{0} / \Delta \nu$. The dominant terms in the expansion of the function $g\left(x, x_{0}\right)$ for $x$ close to $x_{0}$ are

$$
\begin{array}{ll}
g\left(x, x_{0}\right)=\frac{x_{0}}{x}\left[\ln \frac{x_{0}+x}{x_{0}-x}-\frac{1}{x+x_{0}}+\mathrm{e}^{\left(x-x_{0}\right) E \mathrm{i}\left(x_{0}-x\right)}\right. & x<x_{0} \\
g\left(x, x_{0}\right)=\frac{x_{0}}{x}\left[\ln \frac{x+x_{0}}{x-x_{0}}-\frac{1}{x+x_{0}}+\mathrm{e}^{\left(x-x_{0}\right)} E \mathrm{i}\left(x_{0}-x\right)\right] & x>x_{0}
\end{array}
$$

where $E \mathrm{i}$ is the exponential integral function. These functions have the common value

$$
g\left(x, x_{0}\right)=\ln 2 \gamma x_{0}-\frac{1}{2 x_{0}}
$$

where $\ln \gamma$ is Euler's constant at $x=x_{0}$, the absorption edge, as a result of the cancellation of the logarithmic singularity of the first term with that of the exponential integrals. Away from the absorption edge the initial terms alone are accurate to within inverse powers of $\left(x-x_{0}\right)$.

A plot of $n$ calculated from equation (A3) is shown in figure 6. The large peak is absent in the cruder estimate. This peak seems quite reasonable when one reflects that the integral operator is in some ways similar to differentiation - for example, when applied to a symmetrical resonance peak representing $\alpha$, it yields the antisymmetric resonant dispersion curve. Hence, the peak here is due to the rapid increase in $\alpha$ at the ab- 
sorption edge, and its width in some sense is defined by $\Delta \nu$. It can be seen that there is a good correspondence with the shape of the refractive index observed in the laboratory, so that the first approximation to the solution of the integral may be fairly accurate. The difference between the curves disappears with a slight adjustment of the value of $\lambda_{0} \alpha_{0}$.

\section{REFERENCES}

1. Danielson, R. E.; Woolf, N. J.; and Gaustad, J. E.: A Search for Interstellar Ice Absorption in the Infrared Spectrum of Mu Cephei. Astrophys. J., vol. 141, 1965, p. 116.

2. Dressler, K.; and SchnePP, O.: Absorption Spectra of Solid Methane, Ammonia, and Ice in the Vacuum Ultraviolet. J. Chem. Phys., vol. 33, 1960, p. 270.

3. Van De Hulst, H. C.: The Solid Particles in Interstellar Space. Rech. Astron. Obs. Utrecht, vol. 11. pt. 2, 1949.

4. Wickramasinghe, N. C.: Roy. Astron. Soc., Monthlv Notices, vol. 130, 1965, p. 221.

5. Johnson, J. C.; and Terrell, J. R.: J. Opt. Soc. Am., vol. 45, 1955, p. 451.

6. Boggess, A.; and BoRgman, J.: Interstellar Extinction in the Middle Ultraviolet. Astrophys. J., vol. 140, 1964, p. 1638.

\section{DISCUSSION}

Lind: Did you say that, for the best fit, the $300-\AA$ particle size referred to dielectric?

Field: Yes, but I should qualify that remark. At that time, I did not have the infrared data. I don't know what would happen there. In the range between $2200 \AA$ and $8400 \AA$ the data fit to 5 percent. Therefore, I am saying that one can get a rough fit and that is really all that is needed.

Lind: You would need about 10 times larger particles for dielectric fit.

Field: Again, I think that we should be quite precise about the size distribution we are talking about. I am saying that an exponential size distribution with a mean size of $500 \AA$ is actually the best fit.

Greenberg: I doubt that one could get a match. How do you support the 0.05 imaginary part of the refractive index in the visible? The imaginary part of the index of refraction is extremely critical for particle sizes as small as those you have considered.

Field: I quite agree. I don't mean to overemphasize the fit in the visible. We are trying to show here the sensitivity to the size and composition in the UV.

Greenberg: However, if you make a realistic size for dielectric particles to give the extinction in the visible, then the sizes will be such that any spectral characteristics in the ultraviolet will be completely washed out.

Field: That may be. 
O'Dell: Krishna Swamy and I did calculations similar to yours using the wavelength dependence, but we used a value of 0.06 for the imaginary part. When we used the new ultraviolet data and all the infrared data of Johnson, we were able to fit the curve reasonably well out in the infrared by shoving the size parameter-mantle size-down to about $800 \AA$; we still got a reasonable fit in the visible.

Field: I would like to ask again, is one forced to ice, at least in mantles, by Stecher's UV observations? Dr. Wickramasinghe didn't think so.

Wickramasinghe: I did not have available Stecher's observations below $2000 \AA$. These would seem to force one to ice mantles.

Donn: How did you determine the instability of a species as a function of temperature?

Field: Evaporation rates exceed the accretion rate.

Donn: How did you get the accretion rate? Did you assume that all incident oxygen atoms combined with hydrogen to yield $\mathrm{H}_{2} \mathrm{O}$, and similarly for ammonia and methane?

Field: Yes; I simply assumed that each atom of $\mathrm{C}, \mathrm{N}$, and $\mathrm{O}$ that hits the grain, sticks.

Wickramasinghe: Do you assume a constant sticking coefficient for the growth and a size-independent destruction probability?

Field: It is exactly the same formulation that you gave. As for Dr. Donn's question, I think he was referring to the evaporation temperature which I gave in figure 1 . The refractive index effect that Dr. Wickramasinghe and Dr. Stecher are talking about for graphite is a spectroscopic identification similar to the UV extinction effect.

Lind: For the Kramers-Kronig relationship, it seems that you get quite a large discrepancy from what we have. Was this for water?

Field: Ice.

Lind: Was this a plot of the real part of the index of refraction?

Field: Yes; since ice has a sharp absorption edge there must be a large value of $n$.

Lind: The data that we have don't seem to go down so wildly in the ultraviolet. Did you get less than 1 for the real part?

Field: No. The dispersion relation won't permit this. 